九州大学学術情報リポジトリ

Kyushu University Institutional Repository

\title{
ON SMOOTHED PROBABILITY DENSITY ESTIMATION
}

Abdul-al, Khaled I.

University of Petroleum \& Minerals

Geluk, J. L.

University of Petroleum \& Minerals

https://doi.org/10.5109/13406

出版情報: Bulletin of informatics and cybernetics. 23 (3/4), pp.199-208, 1989-03. Research Association of Statistical Sciences

バージョン :

権利関係 : 


\title{
ON SMOOTHED PROBABILITY DENSITY ESTIMATION
}

\author{
By
}

\section{K. I. ABDUL-AL* and J. L. GELUK*}

\author{
Abstract \\ The main object of this paper is to study properties of the estimator

$$
\hat{f}_{n}(x)=\frac{1}{n} \sum_{i=1}^{n} \frac{1}{a_{i}} K\left(\frac{x-X_{i}}{a_{i}}\right)
$$ \\ under the assumption of stationarity of the sequence $\left(X_{n}\right)$.
}

\section{Introduction}

Suppose a sample of observations $X_{1}, X_{2}, \cdots, X_{n}$ is identically distributed with density function $f$. Much research in recent years is concentrated on studying properties of the kernel estimator

$$
\tilde{f}_{n}(x)=\frac{1}{n a_{n}} \sum_{i=1}^{n} K\left(\frac{x-X_{i}}{a_{n}}\right)
$$

where $\left\{a_{n}\right\}, n=1,2, \cdots$ is a given sequence of positive numbers such that $a_{n} \rightarrow 0(n \rightarrow \infty)$ and $K$ is a given kernel. Recently properties of the estimator $\tilde{f}_{n}$ are studied under the assumption of stationarity of the sample. See Masry [10] for the case of a stationary continuous-time process and Castellana and Leadbetter [4] for an approach using $\delta$-sequences. In case of dependence it can be expected that properties of the estimator $\tilde{f}_{n}$ can be improved if the window width is not necessarily the same for each observation, that means the estimator

$$
\hat{f}_{n}(x)=\frac{1}{n} \sum_{i=1}^{n} \frac{1}{a_{i}} K\left(\frac{x-X_{i}}{a_{i}}\right)
$$

is considered. Earlier research concerning $\hat{f}_{n}$ in case of independent observations is done by Devroye [7], Samanta and Mugisha [11], who extended results of Yamato [13], and Davies [5].

One of the results (Theorem 1.3) in this paper is that (in case of dependence) for a suitable choice of the sequence $\left\{a_{n}\right\}$ the variance of the estimator $\hat{f}_{n}$ is smaller than the variance of the usual estimator $\tilde{f}_{n}$. Moreover, under suitable assumptions, the estimator $\hat{f}_{n}$ is asymptotically normal. This is shown in Theorem 2.4 which contains a result about an estimator for $f^{\prime}$ as well. Finally Section 3 contains uniform convergence results, weakly as well as strongly.

\footnotetext{
* University of Petroleum \& Minerals, Dhahran, Saudi Arabia.
} 


\section{Pointwise Consistency}

In this section we determine the asymptotic behaviour of the bias and the variance of the estimator $\hat{f}_{n}$. In order to obtain the asymptotic estimates the sequence $\left\{a_{n}\right\}$ has to be sufficiently smooth. It turns out that regular variation in this case is an appropriate property for the sequence $\left\{a_{n}\right\}$. Lemma 1.1 below contains the basic ingredient for the proof of Theorem 1.3.

Lemma 1.1. (See Bojanic and Seneta [2]) Suppose $\left\{a_{n}\right\} n=1,2, \cdots$ is a sequence of positive numbers. Then

$$
\lim _{n \rightarrow \infty} a_{[n x]} / a_{n}=x^{-\alpha} \quad(x>0) \quad \text { for some } \quad \alpha>-1
$$

if and only if

$$
\lim _{n \rightarrow \infty} \frac{a_{n}}{n} \sum_{j=1}^{n} \frac{1}{a_{j}}=\frac{1}{\alpha+1} \quad \text { with } \quad \alpha>-1
$$

A sequence $\left\{a_{n}\right\}$ satisfying the assumptions of the above lemma is called a regularly varying sequence. For more properties the reader is referred to [9].

COROLlARY 1.2. If $c_{n}>0$ for $n=1,2, \cdots, c_{n} \sim a_{n} \quad(n \rightarrow \infty)$ and the sequence $\left\{a_{n}\right\}$ satisfies the assumptions of the above lemma, then

$$
\lim _{n \rightarrow \infty} \frac{a_{n}}{n} \sum_{j=1}^{n} \frac{1}{c_{j}}=\frac{1}{\alpha+1} .
$$

Theorem 1.3. Suppose $\left\{X_{n}\right\}$ is a stationary sequence of random variables. Let $\hat{f}_{n}^{(r)}$ be defined by

$$
\hat{f}_{n}^{(r)}(x)=\frac{1}{n} \sum_{j=1}^{n} \frac{1}{a_{j}^{r+1}} K^{(r)}\left(\frac{x-X_{j}}{a_{j}}\right), \quad r=0,1 .
$$

Suppose that the following conditions hold:

(i) the sequence $\left\{a_{n}\right\}$ is non-increasing and satisfies (1.1) for some $\alpha>0$.

(ii) $K$ is a symmetric differentiable density such that $\left|K^{(r)}(u)\right| \leqq c, \int K^{(r)}(u)^{2} d u<\infty$ for $r=0,1, \int\left|K^{\prime}(u)\right| d u<\infty$ and there exists $c_{1}$ such that for all $z \ni \Re, t>0$ we have

$$
t\left|K^{(r)}(t z)\right| \leqq c_{1}\left|K^{(r)}(z)\right| \quad \text { for } r=0,1 .
$$

(iii) The joint density $f_{1 j}(x, y)$ of $X_{1}$ and $X_{j}(j=2,3, \cdots)$ is uniformly bounded in $x, y$ and $j$ and satisfies

$$
\sup _{x, y} \sum_{j=2}^{\infty}\left|f_{1 j}(x, y)-f(x) f(y)\right| \leqq M<\infty,
$$

where $f$ is the marginal density of $X_{j}$. Then if $x, y$ are contimuity points of $f$ we have for $r=0,1$

$$
\lim _{n \rightarrow \infty} n a_{n}^{1+2 r} \operatorname{cov}\left(\hat{f}_{n}^{(r)}(x), \hat{f}_{n}^{(r)}(y)\right)= \begin{cases}0 & \text { if } x \neq y \\ \frac{1}{1+(2 r+1) \alpha} f(x) \int K^{(r)}(u)^{2} d u & \text { if } x=y .\end{cases}
$$

Proof. Since $\alpha>0$, we have $a_{n} \rightarrow 0$ as $n \rightarrow \infty$. 


$$
\begin{aligned}
n a_{n}^{1+2 r} \operatorname{cov}\left(\hat{f}_{n}^{(r)}(x), \hat{f}_{n}^{(r)}(y)\right)= & \frac{1}{n} \sum_{j=1}^{n} \frac{a_{n}^{1+2 r}}{a_{j}^{2+2 r}} \operatorname{cov}\left(K^{(r)}\left(\frac{x-X_{j}}{a_{j}}\right), K^{(r)}\left(\frac{y-X_{j}}{a_{j}}\right)\right) \\
& +\frac{a_{n}^{1+2 r}}{n} \sum_{\substack{j \neq k \\
1 \leq j, k \leq n}} \frac{1}{\left(a_{j} a_{k}\right)^{1+r}} \operatorname{cov}\left(K^{(r)}\left(\frac{x-X_{j}}{a_{j}}\right), K^{(r)}\left(\frac{y-X_{k}}{a_{k}}\right)\right) \\
= & : I_{1}+I_{2} .
\end{aligned}
$$

First we estimate $I_{1}$. We have by stationarity

$$
\begin{aligned}
\operatorname{cov}_{1}:= & \operatorname{cov}\left[K^{(r)}\left(\frac{x-X_{j}}{a_{j}}\right), K^{(r)}\left(\frac{y-X_{j}}{a_{j}}\right)\right] \\
= & a_{j} \int K^{(r)}(z) K^{(r)}\left(\frac{y-x}{a_{j}}+z\right) f\left(x-a_{j} z\right) d z \\
& -a_{j}^{2} \int K^{(r)}(z) f\left(x-a_{j} z\right) d z \int K^{(r)}(z) f\left(y-a_{j} z\right) d z .
\end{aligned}
$$

In case $x=y$ we apply Lebesgue's dominated convergence theorem to find

$$
\operatorname{var} K^{(r)}\left(\frac{x-X_{j}}{a_{j}}\right) \sim a_{j} f(x) \int K^{(r)}(z)^{2} d z \quad(j \rightarrow \infty)
$$

for $r=0,1$ at each continuity point $x$ of $f$.

Application of Lemma 1.1 and Corollary 1.2 now gives

$$
I_{1}=\frac{1}{1+(2 r+1) \alpha} f(x) \int K^{(r)}(z)^{2} d z+o(1) \quad \text { as } n \rightarrow \infty
$$

at continuity points $x$ of $f$.

In case $x \neq y$ we can estimate the first terme on the right-hand side in (1.8) as follows. By assumption we have

$$
\begin{aligned}
& \left|a_{j} \int K^{(r)}(z) K^{(r)}\left(\frac{y-x+a_{j} z}{a_{j}}\right) f\left(x-a_{j} z\right) d z\right| \\
& \quad \leqq c_{1}\left|a_{j}^{2} \int K^{(r)}(z) K^{(r)}\left(y-x+a_{j} z\right) f\left(x-a_{j} z\right) d z\right| \\
& \quad \sim c_{1} a_{j}^{2} f(x)\left|K^{(r)}(y-x) \int K^{(r)}(z) d z\right| \quad(j \rightarrow \infty),
\end{aligned}
$$

hence the first term on the right-hand side in $(1.8)$ is $O\left(a_{j}^{2}\right)(j \rightarrow \infty)$. We can estimate the second term by using Lebesgue's dominated convergence theorem. As a consequence $\operatorname{cov}_{1}=O\left(a_{j}^{2}\right)$ as $j \rightarrow \infty$ in case $x \neq y$. Using (1.8), Lemma 1.1 and similar arguments as above, we find $I_{1}=O\left(a_{n}\right)$ as $n \rightarrow \infty$ in case $x \neq y$. Finally we give an estimate for $I_{2}$.

Since the sequence $\left\{a_{n}\right\}$ is nonincreasing, we have

$$
\begin{aligned}
\left|I_{2}\right| \leqq & \frac{a_{n}}{n} \sum_{\substack{j \neq k \\
1 \leqq j, k \leq n}} \frac{1}{a_{j} a_{k}}\left|\operatorname{cov}\left(K^{(r)}\left(\frac{x-X_{j}}{a_{j}}\right), K^{(r)}\left(\frac{y-X_{k}}{a_{k}}\right)\right)\right| \\
\leqq & \frac{a_{n}}{n} \sum_{\substack{j \neq k \\
1 \leqq j, k \leq n}} \iint_{\frac{1}{a_{j} a_{k}}}\left|K^{(r)}\left(\frac{z_{1}}{a_{j}}\right) K^{(r)}\left(\frac{z_{2}}{a_{k}}\right)\right| \mid f_{j k}\left(x-z_{1}, y-z_{2}\right) \\
& -f\left(x-z_{1}\right) f\left(y-z_{2}\right) \mid d z_{1} d z_{2} .
\end{aligned}
$$


From stationarity we have

$$
\begin{aligned}
& \frac{1}{n} \sum_{1 \leqq j<k \leq n}\left|f_{j k}\left(x-z_{1}, y-z_{2}\right)-f\left(x-z_{1}\right) f\left(y-z_{2}\right)\right| \\
& \quad=\sum_{k=2}^{n} \frac{n-k+1}{n}\left|f_{1 k}\left(x-z_{1}, y-z_{2}\right)-f\left(x-z_{1}\right) f\left(y-z_{2}\right)\right| \leqq M,
\end{aligned}
$$

the last inequality being true by assumption (iii). Moreover, a similar inequality holds in case $j>k$.

Combination with (1.4) and (1.10) gives

$$
\begin{aligned}
\left|I_{2}\right| & \leq 2 a_{n} M c_{1}^{2} \iint\left|K^{(r)}\left(z_{1}\right) K^{(r)}\left(z_{2}\right)\right| d z_{1} d z_{2} \\
& =2 a_{n} M c_{1}^{2}\left\{\int\left|K^{(r)}(z)\right| d z\right\}^{2} .
\end{aligned}
$$

REMARK. Note that if $\alpha \in(0,1)$, then $a_{n} \rightarrow 0$ and $n a_{n} \rightarrow \infty \quad(n \rightarrow \infty)$. Hence in this case we have var $\hat{f}_{n}(x) \rightarrow 0(n \rightarrow \infty)$ at all continuity points of $f$ (under the assumptions of Theorem 1.3).

THEOREM 1.4. Suppose the sequence $\left\{a_{n}\right\}$ satisfies the assumptions of Lemma 1.1 with $0<\alpha<\frac{1}{2}$ and $K$ is a symmetric density with $\int|z|^{3} K(z) d z<\infty$. Assume that $X_{1}, X_{2}, \cdots$ are identically distributed with density $f$.

If $\hat{f}_{n}(x)$ is as defined before, $f$ is 3 times differentiable in a neighbourhood of $x$ and $f^{\prime \prime}(x) \neq 0$, then

$$
E \hat{f}_{n}(x)-f(x) \sim \frac{1}{2(1-2 \alpha)} f^{\prime \prime}(x) a_{n}^{2} \int z^{2} K(z) d z \quad(n \rightarrow \infty) .
$$

Proof. Since $K$ is symmetric, we have, using Taylor's theorem

$$
\begin{aligned}
E \hat{f}_{n}(x)-f(x) & =\frac{1}{n} \sum_{j=1}^{n} \int K(z)\left\{f\left(x-a_{j} z\right)-f(x)\right\} d z \\
& =\frac{1}{n} \sum_{j=1}^{n} \int K(z)\left\{-a_{j} z f^{\prime}(x)+\frac{a_{j}^{2} z^{2}}{2} f^{\prime \prime}(x)+O\left(a_{j}^{3} z^{3}\right)\right\} d z \\
& =\frac{1}{2} f^{\prime \prime}(x)\left(\int z^{2} K(z) d z\right) \frac{1}{n} \sum_{j=1}^{n} a_{j}^{2}(1+o(1)) \quad(n \rightarrow \infty) .
\end{aligned}
$$

Application of Lemma 1.1 finally gives (1.12).

Under the assumptions of Theorem 1.3 and Theorem 1.4 we find that the mean square error is equal to

$$
\begin{aligned}
E\left\{\hat{f}_{n}(x)-f(x)\right\}^{2} & =\frac{1}{1+\alpha} f(x) \int K^{2}(z) d z \frac{1}{n a_{n}} \\
& +\frac{1}{4(1-2 \alpha)^{2}} f^{\prime \prime}(x)^{2}\left(\int z^{2} K(z) d z\right)^{2} a_{n}^{4}+o\left(\frac{1}{n a_{n}}\right)+o\left(a_{n}^{\prime}\right) \quad(n \rightarrow \infty) .
\end{aligned}
$$

It is easily seen that in case $a_{n}=c n^{-\alpha}$, the optimal choice for $\alpha$ is $1 / 5$. In that case we have 


$$
E\left\{\hat{f}_{n}(x)-f(x)\right\}^{2}=\left\{\frac{5}{6} f(x)\left(\int K^{2}(z) d z\right) c^{-1}+\frac{25}{36} f^{\prime \prime}(x)^{2}\left(\int z^{2} K(z) d z\right)^{2} c^{4}\right\}^{n^{-4 / 5}}
$$

The optimal choice of $c$ then finally depends upon the kernel $K$. Depending upon the kernel and the values of $f(x)$ and $f^{\prime \prime}(x)$ the result may be better or worse than the usual estimate.

\section{Asymptotic Normality}

In this section we prove asymptotic normality of the estimator $\hat{f}_{n}$ in case the sequence of random variables $\left\{X_{n}\right\}$ satisfies an array form of the strong mixing condition.

Definition 2.1. The stationary sequence $\left\{X_{n}\right\}$ has strong mixing coefficients $\alpha_{n, l}$ if

$$
\alpha_{n, l}:=\max _{1 \leqq i \leqq n-l} \sup _{\substack{A \in \sigma(0, i) \\ B \in \sigma(i+l, n)}}|P(A B)-P(A) P(B)|
$$

for $1 \leqq l \leqq n-1$, where $\sigma(i, j)$ is the $\sigma$-field generated by $\left\{X_{k} ; 1<k \leqq j\right\}$.

We need two lemmas. The first is due to Volkonskii and Rozanov [12].

Lemma 2.2. Suppose $X_{1}, X_{2}, \cdots, X_{m}$ are random variables measurable with respect to $\sigma\left(i_{1}, j_{1}\right), \sigma\left(i_{2}, j_{2}\right), \cdots, \sigma\left(i_{m}, j_{m}\right)$ respectively, where $0 \leqq i_{1}<j_{1}<i_{2}<\cdots<i_{m}<j_{m} \leqq n$, $i_{k+1}-j_{k} \geqq l \geqq 1$ and $\left|X_{k}\right| \leqq 1,1 \leqq k \leqq m$. Then

$$
\left|E\left(\prod_{k=1}^{m} X_{k}\right)-\prod_{k=1}^{m} E X_{k}\right| \leqq 16(m-1) \alpha_{n, l}
$$

where $\alpha_{n, l}$ is as in (2.1).

LEMMA 2.3. If the sequence $\left\{a_{n}\right\}$ satisfies (1.1) and $\tau=\tau(n), m=m(n)$ are such that $\tau(n) m(n) \sim n(n \rightarrow \infty)$, then $\sum_{k=1}^{m} 1 / a_{k \tau} \sim n /(1+\alpha) \tau a_{n}(n \rightarrow \infty)$

Proof. Define $f(x):=1 / a_{[x]}$. Since the function $f$ satisfies $f(t x) / f(t) \rightarrow x^{+\alpha}(t \rightarrow \infty)$ for $x>0$, convergence is uniform on compact intervals of $(0, \infty)$ (see de Bruijn [3]). Moreover $\int_{1}^{x} f(s) d s \sim x f(x) /(1+\alpha)(x \rightarrow \infty)$. As a consequence

$$
\begin{aligned}
\sum_{k=1}^{m} 1 / a_{k \tau} & =\int_{1}^{m+1} f([s] \tau) d s \sim \int_{1}^{m+1} f(s \tau) d s \sim \int_{0}^{n / \tau} f(s \tau) d s \\
& =\frac{1}{\tau} \int_{0}^{n} f(u) d u \sim n /(1+\alpha) \tau a_{n} \quad(n \rightarrow \infty) .
\end{aligned}
$$

THEOREM 2.4. Suppose the assumptions of Theorem 1.3 are satisfied. Assume that there exists a sequence of integers $k_{n}(n \geqq 1)$ for which

$$
\left(n / a_{n}\right)^{1 / 2} \alpha_{n, k_{n}} \longrightarrow 0 \text { and } k_{n}=o\left(n a_{n}\right)^{1 / 2} \quad(n \rightarrow \infty) .
$$

Then if $\alpha \in(0,1)$ (see 1.1)) at each continuity point $x$ of $f^{(r)}$

where

$$
\left(\hat{f}_{n}^{(r)}(x)-E \hat{f}_{n}^{(r)}(x)\right) c_{0}\left(n a_{n}^{2 r+1}\right)^{1 / 2},
$$

$$
c_{0}=c_{0, r}=\left\{\frac{1}{1+(2 r+1) \alpha} f(x) \int K^{(r)}(z)^{2} d z\right\}^{-1 / 2}
$$


has the standard normal limiting distribution for $r=0$ and $r=1$.

PRoOF. From (2.3) it follows that there exists a sequence $\lambda_{n}$ such that $\lambda_{n} \rightarrow \infty$ $(n \rightarrow \alpha), \lambda_{n} k_{n}=o\left(n a_{n}\right)^{1 / 2}$ and $\lambda_{n}\left(n / a_{n}\right)^{1 / 2} \alpha_{n, k_{n}} \rightarrow 0(n \rightarrow \infty)$.

We write the expression in (2.4) as a sum of $m$ blocks of length $\tau$ (the large blocks $\left.\left(S_{1}\right)\right)$ and $m$ blocks of length $\tau^{\prime}$ (the small blocks $\left(S_{2}\right)$ ), where $m=\left[n /\left(\tau+\tau^{\prime}\right)\right]$, and a resulting block. Define $\tau, \tau^{\prime}$ by $\tau=\left[\lambda_{n}^{-1}\left(n a_{n}\right)^{1 / 2}\right]$ and $\tau^{\prime}=k_{n}$. Note that $\tau, \tau^{\prime}$ depend on $n, n a_{n} \rightarrow \infty$ since $\alpha \subseteq(0,1)$ and

$$
\tau=o\left(n a_{n}\right)^{1 / 2}, \quad \tau^{\prime}=o(\tau) \text { and } \frac{n}{\tau} \alpha_{n, \tau^{\prime}} \longrightarrow 0 \quad(n \rightarrow \infty) .
$$

Now for

$$
\begin{aligned}
\left(\hat{f}_{n}^{(r)}(x)-E \hat{f}_{n}^{(r)}(x)\right) c_{0} \sqrt{a_{n}^{2 r+1} n} & =\sum_{i=1}^{n} \frac{a_{n}^{(2 r+1) / 2}}{n^{1 / 2}} \frac{c_{0}}{a_{i}^{r+1}}\left\{K^{(r)}\left(\frac{x-X_{i}}{a_{i}}\right)-E K^{(r)}\left(\frac{x-X_{i}}{a_{i}}\right)\right\} \\
& =\sum_{i=1}^{n} Y_{i} .
\end{aligned}
$$

We write

where

$$
\sum_{i=1}^{n} Y_{i}=\sum_{k=1}^{m} \sum_{j \in I_{k, n}} Y_{j}+\sum_{k=1}^{m} \sum_{j \in I_{k, n}^{\prime}} Y_{j}+\sum_{j \in I_{n}^{\prime \prime}} Y_{j}=: S_{1}+S_{2}+S_{3}
$$

and

$$
\begin{aligned}
& A_{k, n}=\left\{(k-1)\left(\tau+\tau^{\prime}\right)+1, \cdots,(k-1)\left(\tau+\tau^{\prime}\right)+\tau\right\} \\
& A_{k, n}^{\prime}=\left\{(k-1)\left(\tau+\tau^{\prime}\right)+\tau+1, \cdots, k\left(\tau+\tau^{\prime}\right)\right\}
\end{aligned}
$$

$$
A_{n}^{\prime \prime}=\left\{m\left(\tau+\tau^{\prime}\right)+1, \cdots, n\right\} .
$$

First we show that $\operatorname{var} S_{2} \rightarrow 0$ as $n \rightarrow \infty$. Using (1.9) we find

$$
\begin{aligned}
\operatorname{var} S_{2} \leqq \frac{c_{0}^{2} a_{n}^{2 r+1}}{n} & \sum_{k=1}^{m} \sum_{j \in 1_{k, n}^{\prime}} \frac{c_{1}}{a_{j}^{2 r+1}} \\
& \quad+\frac{2 c_{0}^{2} a_{n}}{n} \sum_{1 \leqq j<k \leqq n} \frac{1}{a_{j} a_{k}}\left|\operatorname{cov}\left(K^{(r)}\left(\frac{x-X_{j}}{a_{j}}\right), K^{(r)}\left(\frac{x-X_{k}}{a_{k}}\right)\right)\right|
\end{aligned}
$$

where $c_{1}>f(x) \int K^{(r)}(z)^{2} d z$ is a constant.

Since $\sum_{j \in l_{k, n}^{\prime}} \frac{1}{a_{j}^{2 r+1}} \sim \frac{\tau^{\prime}}{a_{k \tau}^{2 r+1}}(n \rightarrow \infty)$, Lemma 2.3 together with (1.11) show that

$$
\operatorname{var} S_{2} \leqq c_{2} \frac{a_{n}^{2 r+1}}{n} \cdot \tau^{\prime} \cdot \frac{n}{\tau a_{n}^{2 r+1}}+c_{3} a_{n}=c_{2} \tau^{\prime} / \tau+c_{3} a_{n} \longrightarrow 0
$$

as $n \rightarrow \infty$, where $c_{2}$ and $c_{3}$ are constants.

Since $E S_{2}=0$ this implies $P\left(\left|S_{2}\right|>\varepsilon\right) \leqq \operatorname{var} S_{2} / \varepsilon^{2} \rightarrow 0$ as $n \rightarrow \infty$. Similarly we find that $S_{3}$ tends to zero in probability. As a consequence, the asymptotic distribution of $\sum_{i=1}^{n} Y_{i}$, if it exists, is the same as for $S_{1}$.

We claim that the asymptotic distribution (if it exists) of $S_{1}$ is the same as the distribution of $S_{1}^{\prime}=\sum_{k=1}^{m} \gamma_{k}$ where $\gamma_{k}=\sum_{j \in A_{k}, n} Y_{j}$ and the $\gamma_{k}$ 's are independent. Indeed, by Lemma 2.2, $\mid E\left(\exp \left(\right.\right.$ it $\left.\left.\sum_{k=1}^{m} \gamma_{k}\right)\right)-\prod_{k=1}^{m} \exp \left(\right.$ it $\left.\gamma_{k}\right) \mid$ is bounded by $16(m-1) \alpha_{n, \tau^{\prime}} \sim 16 \frac{n}{\tau} \alpha_{n, \tau^{\prime} \rightarrow 0}$ 
$(n \rightarrow \infty)$ (see $(2.6))$.

Finally we prove that $S_{1}^{\prime} \stackrel{D}{\longrightarrow} N$, where $N$ is the standard normal distribution. First we show that $\operatorname{var} S_{1}^{\prime} \rightarrow 1$ as $n \rightarrow \infty$.

$$
\operatorname{var} \gamma_{k}=\operatorname{var} \sum_{j \in I_{k, n}} Y_{j}=\sum_{j \in I_{k}, n} \operatorname{var} Y_{j}+\sum_{i, j=I_{k}, n}\left(\operatorname{cov} Y_{j}, Y_{i}\right)=T_{1, k}+T_{2, k} .
$$

Note that by (1.9) and Lemma 2.3 we have

$$
\begin{aligned}
T_{1, k} & \sim \sum_{j \in 1_{k, n}} \frac{a_{n}^{2 r+1}}{n} \frac{c_{0}^{2}}{a_{j}^{2 r+2}} a_{j} f(x) \int K^{(r)}(z)^{2} d z \\
& \sim \frac{1}{1+(2 r+1) \alpha} \frac{c_{0}^{2} a_{n}^{2 r+1}}{n} f(x) \int K^{(r)}(z)^{2} d z\left(\frac{k \tau}{a_{k=}^{2 r+1}}-\frac{(k-1) \tau}{a_{(k-1) \tau}^{2 r+1}}\right) .
\end{aligned}
$$

Moreover, with $I_{2}$ as defined in (1.7), we have

$$
\sum_{k=1}^{m} T_{2, k} \leqq\left|I_{2}\right|=O\left(a_{n}\right) \quad(n \rightarrow \infty) \quad(\text { as in }(1.11)) .
$$

Summation over $k \in\{1,2, \cdots, m\}$ then gives

$$
\operatorname{var} S_{1}^{\prime} \sim \frac{a_{n}^{2 r+1}}{n} \frac{m \tau}{a_{m \tau}^{2 r+1}} \longrightarrow 1 \quad \text { as } n \rightarrow \infty .
$$

Finally we verify that the Lindeberg condition

$$
\sum_{k=1}^{m} \frac{1}{S_{m}^{2}} E\left\{\gamma_{k}^{2} I\left(\left|\gamma_{k}\right| \geqq \varepsilon S_{m}\right)\right\} \longrightarrow 0 \quad \text { for each } \quad \varepsilon>0
$$

where $S_{m}^{2}=$ var $S_{1}^{\prime}$, is satisfied.

By the definition of $Y_{j}$ (see (2.7)) and assumption (ii) in Theorem 1.3 we have

$$
\left|\gamma_{k}\right| \leqq \sum_{j \in I_{k, n}}\left|Y_{j}\right| \leqq \sum_{j=(k-1)=}^{k\left(\xi+z^{\prime}\right)} \frac{\sqrt{a_{n}^{2 r+1}}}{\sqrt{\bar{n}}} \frac{2 c c_{0}}{a_{j}^{r+1}} .
$$

In view of Lemma 1.1 the last expression is asymptotic to

$$
c^{\prime} \frac{a_{n}^{(2 r+1) / 2}}{\sqrt{n}}\left\{\frac{k \tau}{a_{k \tau}^{r+1}}-\frac{(k-1) \tau}{a_{(k-1) \tau}^{r+1}}\right\}=O\left(\frac{\tau a_{n}^{(2 r+1) / 2}}{\sqrt{n} a_{k \tau}^{r+1}}\right) \quad(n \rightarrow \infty)
$$

where $c^{\prime}$ is a constant. Since the sequence $\left\{a_{n}\right\}$ is non-increasing and $n a_{n} \rightarrow \infty$, we have $O\left(\tau a_{n}^{(2 r+1) / 2} / \sqrt{n} a_{k \tau}^{r+1}\right)=o(1)$, so that $P\left(\left|\gamma_{k}\right|>\varepsilon\right)=0$ for all $n$ sufficiently large. This finishes the proof of (2.9) since $S_{\bar{m}}^{2} \rightarrow 1$.

\section{Uniform Convergence}

In this section a uniform error measure, namely

$$
W_{n}^{(r)}:=\sup _{x \in R}\left|\hat{f}_{n}^{(r)}(x)-f^{(r)}(x)\right|
$$

is discussed. Under suitable conditions regarding the strong mixing coefficients $\alpha_{n, l}$ and the sequence $\left\{a_{n}\right\}$ and restrictions on the kernel $K$, it will be shown that $W_{n}^{(r)}$ 
converges to 0 weakly as well as strongly. We define the functions $\phi, k$ and $\dot{\phi}_{n}$ by

$$
\begin{aligned}
& \phi(t)=\int e^{i t x} f(x) d x \\
& k(t)=\int e^{i t x} K(x) d x
\end{aligned}
$$

and

$$
\dot{\phi}_{n}(t)=\frac{1}{n} \sum_{j=1}^{n} e^{i t x} j
$$

THEOREM 3.1. Assume $\left\{X_{n}\right\}$ is a sequence of stationary random variables. Suppose the following conditions hold for some integer $r \geqq 0$.

(i) $K$ is symmetric.

(ii) $k$ is non-increasing on $\boldsymbol{R}^{+}$and $t^{m} k(t) \subseteq L^{1}(-\infty, \infty)$ for $0 \leqq m \leqq r$,

(iii) the sequence $\left\{a_{n}\right\}$ is non-increasing, $a_{n} \rightarrow 0(n \rightarrow \infty)$ and $n a_{n}^{2(r+1)} \rightarrow \infty(n \rightarrow \infty)$,

(iv) the strong mixing coefficients $\alpha_{n, j}$ (see (2.1)) satisfy the condition

$$
\varlimsup_{n \rightarrow \infty} \sum_{j=1}^{n-1} \alpha_{n, j}<\infty
$$

(v) $f^{(m)}(x)$ is bounded and continuous for $0 \leqq m \leqq r$.

Then $W_{n}^{(r)} \stackrel{P}{\rightarrow} 0$ as $n \rightarrow \infty$.

Proof. Similar to the proof of Theorem 3.8 in Abdul-Al and Siddiqui [1]. Since $k \in L^{1}\left(-\infty, \infty\right.$ ) we have $K(x)=(2 \pi)^{-1} \int e^{-i t x} k(t) d t$ (see e.g. Feller [8] XV. 4, Lemma 2). In view of assumption (ii) we may differentiate $r$ times to find

$$
K^{(r)}(x)=(2 \pi)^{-1}(-i)^{r} \int t^{r} e^{-i t x} k(t) d t .
$$

By substitution in (1.3) we find

$$
\hat{f}_{n}^{(r)}(x)=(2 \pi n)^{-1} \int_{j=1}^{n}(-t)^{r} e^{-i t\left(x-x_{j}\right)} k\left(a_{j} t\right) d t .
$$

Hence we have

$$
V_{n}:=\sup _{x \in \boldsymbol{R}}\left|\hat{f}_{n}^{(r)}(x)-E \hat{f}_{n}^{(r)}\right| \leqq \frac{1}{2 \pi n} \int\left|t^{r} \sum_{j=1}^{n}\left\{e^{i t x_{j}}-\boldsymbol{\phi}(t)\right\} k\left(a_{j} t\right)\right| d t .
$$

Since $a_{n}$ is non-increasing and $k$ is non-increasing, this implies

$$
V_{n} \leqq \frac{1}{2 \pi} \int|t|^{r}\left|\phi_{n}(t)-\phi(t)\right|\left|k\left(a_{n} t\right)\right| d t .
$$

Hence, by Fubini's theorem and Schwarz' inequality,

$$
\left.E V_{n} \leqq \frac{1}{2 \pi} \int\left\{\sigma^{2}\left(\phi_{n}(t)\right)\right\}^{1 / 2} \mid t^{r} k\left(a_{n} t\right)\right\} d t
$$

By stationarity and Lemma 2.2 we have 


$$
\begin{aligned}
\sigma^{2}\left(\phi_{n}(t)\right) & =\frac{1}{n} E\left|e^{i t x}-\phi(t)\right|^{2}+\frac{2}{n^{2}} \sum_{j=2}^{n}(n-j+1) E\left[e^{i t\left(x_{1}-x_{j}\right)}-E e^{i t x_{1}} E e^{i t x_{j}}\right] \\
& \leqq \frac{4}{n}+\frac{32}{n} \sum_{j=1}^{n-1} \alpha_{n, j} .
\end{aligned}
$$

Combination with (3.5) gives

$$
E V_{n} \leqq \frac{2}{\pi n^{1 / 2} a_{n}^{r+1}}\left(\int\left|t^{r} k(t)\right| d t\right)\left(1+8 \sum_{j=1}^{n-1} \alpha_{n, j}\right)^{1 / 2} \longrightarrow 0 \quad \text { as } n \rightarrow \infty \text {, hence } V_{n} \stackrel{P}{\rightarrow} 0
$$

Since $f(x)$ and its first $r$ derivatives are bounded and continuous and $\lim _{|u| \rightarrow \infty} K^{(m)}(u)=0$ for $0 \leqq m \leqq r$ (this follows from (3.2) by application of the Riemann-Lebesgue Lemma), we have

$$
E \hat{f}_{n}^{(r)}(x)=\frac{1}{a_{j}^{r+1}} \int K^{(r)}\left(\frac{x-u}{a_{j}}\right) f(u) d u=\frac{1}{a_{j}} \int K\left(\frac{x-u}{a_{j}}\right) f^{(r)}(u) d u
$$

Application of Lemma 1 in Yamato [13] then gives

$$
\sup _{x \in R}\left|E \hat{f}_{n}^{(r)}(x)-f^{(r)}(x)\right| \longrightarrow 0 \quad \text { as } n \rightarrow \infty .
$$

Combination with (3.6) finishes the proof.

In the remainder of this section we discuss strong uniform convergence. We omit the proof which is similar to the proof of theorem 3.9 in Abdul-Al and Siddiqui [1] (using Lemma 4.1 in Davydov [6]).

THEOREM 3.2. Suppose $\left\{X_{n}\right\}$ is a sequence of stationary random variables satisfying the following conditions. Suppose conditions (i) and (ii) of Theorem 3.1 hold and

(i) $f$ is uniformly continuous,

(ii) the sequence $\left\{a_{n}\right\}$ satisfies $a_{n} \rightarrow 0(n \rightarrow \infty)$ and

$$
\sum_{n=1}^{\infty} \frac{1}{n^{2} a_{n}^{4(r+1)}}<\infty,
$$

(iii) $x^{r} K(x) \rightarrow 0(x \rightarrow \infty)$, and

(iv) $\sum_{j=1}^{\infty}\left(\sup _{n} \alpha_{n, j}\right)<\infty$.

Then

$$
\sup _{s \in R}\left|\hat{f}_{n}^{(r)}(x)-f^{(r)}(x)\right| \stackrel{W . P .1}{\longrightarrow} 0 \quad \text { as } n \rightarrow \infty \text {. }
$$

\section{References}

[1] Abdel-Al, K.I. and Siddiqu, M. M.: Density estimation for uniform mixing process, Bull. Inf. Cyb., 22 (1986), 79-93.

[2] Bojanic, R. and Seneta, E.: A unified theory of regularly iarying sequences, Math. Z., 134 (1973), 91-106.

[3] De Bruljn, N.G.: Pairs of slowly oscillating functions occurring in asymptotic problems concerning the Laplace transform, Nw. Arch. v. Wisk., 7 (1959), 20-26.

[4] Castellana, J.V. and LeadbetTer, M.R.: On smoothed probability density estimation for stationary processes, Stoch. Proc. and Appl., 21 (1986), 179-198. 
[5] Davies, H.I.: Strong consistency of a sequential estimator of a p.d.f., Bull. Math. Stat., 15 (1973), 49-53.

[6] Davydov, Yu.A.: Convergence of distribution generated by stationary stochastic processes, Theory Prob. Applications, 13 (1968), 691-696.

[7] Devroye, Luc P.: On the pointwise and the integral convergence of recursive kernel esti. mates of probability densities, Utilitas Math., 15 (1975), 113-128.

[8] Feller, W.: An introduction to probability theory and its applications, Wiley, 2 (1971).

[9] GeLuK, J.L. and DE HAA., L.: Regular variation, extensions and Tauberian theorems, CWI tract 40, Amsterdam, (1987).

[10] MASRY, E.: Probability density estimation from sampled data, IEEE Trans. Inf. Theory. IT, 29 (1983), 696-709.

[11] Samanta, M. and Mugisha, R.X.: On a class of estimates of the probability density function and mode based on a random number of observations, Calcutta Stat. Assoc. Bull., (1981), 23-40.

[12] Volkonskil, V.A. and RozAnov, Y.A.: Some limit theorems for random functions, Theory Prob. Appl., 4 (1959), 178-197.

[13] Yamato, H.: Sequential estimation of a continuous probability density function and mode, Bull. Math. Stat., 14 (1971), 1-12.

Received January 7, 1988

Revised March 18, 1988

Communicated by T. Kitagawa 